\title{
On the Nature of Space Fluctuations of Solutions of Dissipative Partial Differential Equations
}

\author{
Michele V. Bartuccelli \\ Department of Mathematics, University of Surrey, Guildford, GU2 7XH, UK
}

January 14, 2019

\begin{abstract}
In this work we have analysed the nature of space fluctuations in dissipative Partial Differential Equations (PDEs). By taking a well known and much investigated dissipative PDE as our representative, namely the Swift-Hohenberg Equation, we estimated in an explicit manner the values of the crest factor of its solutions. We believe that the crest factor, namely the ratio between the sup-norm and the $L^{2}$ norm of solutions, is a suitable and proper measure of space fluctuations in solutions of dissipative PDEs. In particular it gives some information on the nature of "soft" and

"hard" fluctuations regimes in the flows of dissipative PDEs.
\end{abstract}

Short title: Crest Factor and Space Fluctuations of PDEs.

Keywords: Dissipative Partial Differential Equations; Best Constants; Analysis of Solutions; Crest Factor.

Classification numbers: 35B40, 35B45, 35G20, 35K25, 46E20, 46E35

\section{Introduction}

One of the outstanding open problems of applied mathematics is understanding the nature of turbulence. There is a vast literature on this very intricate and deep problem. Here we can just mention some books devoted to this fascinating subject $[1,2]$. An essential feature of turbulence is its space time intermittent behaviour characterised by random fluctuations in the turbulent solutions of dissipative partial differential equations (PDEs); the most famous and celebrated example of dissipative PDEs are the Navier-Stokes equations of fluid dynamics.

In this work we will endeavour to understand a possible way to "measure" the space fluctuations of solutions of dissipative PDEs. The comprehension of fluctuations is naturally linked to the nature of turbulence and represents one of its most important hallmarks. The method we use in this work for capturing space fluctuations of dissipative PDEs is functional analysis. First we we will compute in an explicit and accurate way some estimates for some Sobolev norms of solutions of some typical dissipative PDEs in several space dimensions. Then we use these estimates to introduce an important concept in the analysis of the behaviour of solutions of dissipative PDEs, namely the so-called "crest factor", which is defined as the ratio between the sup-norm and the $L^{2}$ norm of solutions. As its name expressively suggests, the crest factor measures the fluctuations and distorsions between the amplitude of the sup-norm and the $L^{2}$ norm of the solutions in PDEs. It is in fact widely used in real experiments made in wind tunnels for understanding some of the hallmarks of turbulence in fluid dynamics. The larger the crest factor, the larger the potential space fluctuations. As it is well known, measuring large and intermittent space fluctuations, is one of the fundamental aspects for understanding the nature of turbulence.

In this work, in order to illustrate how to compute in an operative way the crest factor, we take as our standard example of dissipative PDE (a version of) the relatively well understood Swift-Hohenberg Equation (SHE) [13]:

$$
u_{t}=-(\Delta)^{2} u-\Delta u+\lambda u-u^{2 p+1},
$$


where $\Delta$ is the Laplacian, with a flat domain $\Omega=[0, L]^{d}$, with $L>0, t>0$ and $p$ is a positive integer. It is subject to the initial condition $u(\vec{x}, 0)=u_{0}(\vec{x})$ in $d=1,2,3$ space dimensions and periodic boundary conditions on the boundary of $\Omega$. The parameter $\lambda$ is a positive constant.

In the rest of this work we will compute various Sobolev norms of the solutions of the SHE and hence we will estimate their crest factor. We will then discuss the implications deriving from our estimates of the crest factor in space dimension $d=1,2,3$ in the conclusion and we provide some open problems.

\section{Explicit Estimates of the Crest Factor of the SHE}

In this section we obtain estimates on various Sobolev norms of the solutions and their spatial derivatives. We then use these estimates for computing the time averaged crest factor. For explicit and accurate estimates regarding the time-asymptotic behaviour of the sup-norm of solutions for the case $p=1$ see [5].

Before commencing our analysis, for the sake of the reader, let us first give a brief functional analysis settings and notations [12]. Denote by $\Omega=[0, L]^{d}$ the $d$-dimensional torus; for any scalar function $\phi(x)$ with $x \in \Omega$ let $\|\phi\|_{p}^{p}=\int_{\Omega}|\phi(x)|^{p} \mathrm{~d} x$ be the norm associated with the Banach space of functions in $\Omega$ and we also define the $L^{\infty}$ norm as

$$
\|\phi\|_{\infty}=\sup _{x \in \Omega}|\phi(x)|
$$

For $p=2$ we denote by $L^{2}(\Omega)$ the Hilbert space of $\Omega$-periodic functions $\phi$ with $\|\phi\|_{2}<+\infty$. Given a multi-index $\vec{n}=\left(n_{1}, n_{2}, \ldots, n_{d}\right)$, with all the $n_{i}$ non-negative integers, let $|\vec{n}|=n_{1}+\ldots+n_{d}$ and

$$
D^{\vec{n}}:=\frac{\partial^{|\vec{n}|}}{\partial x_{1}^{n_{1}} \partial x_{2}^{n_{2}} \cdots \partial x_{d}^{n_{d}}},
$$

and let

together with

$$
J_{n}:=\left\{\phi: \int_{\Omega}\left(D^{\vec{n}} \phi\right)^{2} \mathrm{~d} x<+\infty \text { for all } \vec{n} \text { such that }|\vec{n}|=n\right\}
$$

$$
\|\phi\|_{2}^{2}:=\sum_{\substack{n_{1}, \ldots, n_{d} \geq 0 \\ n_{1}+\ldots+n_{d}=n}} \frac{n !}{n_{1} ! \cdot n_{d} !}\left\|D^{\vec{n}} \phi\right\|_{2}^{2} .
$$

Let us also first mention what we mean by the crest factor of a solution of a dissipative PDE [3].

The crest factor (also known as the peak to average ratio) is defined as the ratio between the $L^{\infty}$ norm and the $L^{2}$ norm of a solution of any partial differential equation, namely

$$
C_{f}:=L^{\frac{d}{2}} \frac{\|u\|_{\infty}}{J_{0}^{\frac{1}{2}}} .
$$

One can see that it is (by definition) dimensionless and it contains important information on the "distorsions" between the sup-norm (the amplitude) and the $L^{2}$ norm of the solution. The ideal result would be to have a time-pointwise estimate of $C_{f}$. However this is very difficult due essentially to the non-linearity of the equation. So in this work we will compute the time-average of the quotient between the $L^{\infty}$ norm and the $L^{2}$ norm of the solution, namely $\left\langle L^{\frac{d}{2}}\|u\|_{\infty} / J_{0}^{\frac{1}{2}}\right\rangle$.

First of all let us derive sharp estimates for the $\|u\|_{\infty}$ of typical solutions $u(x, t)$. Note that in general we cannot assume that the solutions of our equation have zero-mean. Hence we have to "carry along" the mean value of our solutions. Thus suppose that $\int_{\Omega} u(x) \mathrm{d} x \neq 0$ and $u(x)=u^{*}+u^{\prime}(x)$, where $u^{*}=$ const $\neq 0$ and $\int_{\Omega} u^{\prime}(x) \mathrm{d} x=0$. Then using the inequality

$$
\left|u^{*}\right|=L^{-d}\left|\int_{\Omega} u(x) \mathrm{d} x\right| \leq L^{-\frac{d}{2}} J_{0}^{\frac{1}{2}}
$$


and defining $J_{0}^{\prime}:=\left\|u^{\prime}\right\|_{2}^{2}$, we obtain [7]

$$
\|u\|_{\infty} \leq\left|u^{*}\right|+\left\|u^{\prime}\right\|_{\infty} \leq L^{-\frac{d}{2}} J_{0}^{\frac{1}{2}}+c(n)\left(J_{0}^{\prime}\right)^{\frac{2 n-d}{4 n}} J_{n}^{\frac{d}{4 n}} .
$$

with $n>1 / 2$ and $c(n)$ a suitable constant, where we have used a Gagliardo-Nirenberg inequality to obtain the estimate on $\left\|u^{\prime}\right\|_{\infty}$. By substituting $u=1$ in (4) we see that the constant $L^{-\frac{d}{2}}$ is sharp. Therefore we obtain the following estimate

$$
\frac{\|u\|_{\infty}}{J_{0}^{\frac{1}{2}}} \leq \frac{\left|u^{*}\right|+\left\|u^{\prime}\right\|_{\infty}}{J_{0}^{\frac{1}{2}}} \leq L^{-\frac{d}{2}}+\frac{\left\|u^{\prime}\right\|_{\infty}}{J_{0}^{\frac{1}{2}}} .
$$

Hence by using (5) we obtain

$$
\frac{\|u\|_{\infty}}{J_{0}^{\frac{1}{2}}} \leq L^{-\frac{d}{2}}+c(n)\left(\frac{J_{n}}{J_{0}}\right)^{\frac{d}{4 n}}\left(\frac{J_{0}^{\prime}}{J_{0}}\right)^{\frac{2 n-d}{4 n}} .
$$

Thus our estimate for the crest factor is obtained by taking the time-average

$$
\widetilde{C}_{f}:=\left\langle L^{\frac{d}{2}} \frac{\|u\|_{\infty}}{J_{0}^{\frac{1}{2}}}\right\rangle .
$$

It is useful to concentrate on the "pure" distortion between the sup-norm and the $L^{2}$ norm for nonconstant solutions (note that of course purely time dependent functions or constants have crest factor equal to 1). Bearing this in mind one obtains

$$
\widetilde{C}_{f}=1+\bar{C}_{f}, \quad \bar{C}_{f}:=\left\langle L^{\frac{d}{2}} \frac{\left\|u^{\prime}\right\|_{\infty}}{J_{0}^{\frac{1}{2}}}\right\rangle \leq c(n) L^{\frac{d}{2}}\left\langle\left(\frac{J_{n}}{J_{0}}\right)^{\frac{d}{4 n}}\right\rangle,
$$

where the last bound follows noting that $J_{0}^{\prime} \leq J_{0}$. Note that, since one has trivially $\bar{C}_{f}=0$ if $u(x, t)$ does not depend on $x$, in order to estimate the crest factor we may assume in the following that $u^{\prime} \neq 0$. Hence $J_{n}>0$ for all $n \geq 0$.

Bearing in mind all of the above we can now start our analysis of (a version of) the SHE on the $d$-dimensional flat torus with domain $\Omega=[0, L]^{d}$ with $d=1,2,3$.

\section{Analysis in space dimension one}

We begin with one spatial dimension $(d=1)$, namely we study

$$
u_{t}=-u_{x x x x}-u_{x x}+\lambda u-u^{2 p+1},
$$

with periodic boundary conditions on $\Omega=[0, L]$. First we estimate the evolution of the time-dependent quantity $J_{0}(t)=\int_{\Omega} u^{2}(x, t) \mathrm{d} x$. By differentiating it with respect to time one finds

$$
\frac{1}{2} \dot{J}_{0}=-J_{2}+J_{1}+\lambda J_{0}-\int_{\Omega}(u)^{2 p+2} \mathrm{~d} x .
$$

First note that (8) holds true in every spatial dimension. Also we observe that

$J_{1} \leq\left(J_{2} J_{0}\right)^{\frac{1}{2}} \leq \frac{1}{2}\left(J_{0}+J_{2}\right)$ and that $-\int_{\Omega}(u)^{2 p+2} \mathrm{~d} x \leq-\frac{J_{0}^{p+1}}{L^{p d}}$, with $d$ the spatial dimension. Collecting and simplifying we have

$$
\frac{1}{2} \dot{J}_{0} \leq-\frac{J_{2}}{2}+\frac{2 \lambda+1}{2} J_{0}-\frac{J_{0}^{p+1}}{L^{p d}} .
$$

We now take $d=1$, and we first divide the above by $J_{0}$ and then take its time average obtaining

$$
\left\langle\frac{J_{2}}{J_{0}}\right\rangle \leq 2 \lambda+1
$$


Note that the time average of the term $\frac{1}{2} \frac{\dot{J}_{0}}{J_{0}}$ vanishes as $J_{0}>0$ is bounded above and also below away from zero because $\lambda>0$. In fact we choose $\lambda$ larger than the first eigenvalue in the linearised equation, namely we take $\lambda>\left(\frac{2 \pi}{L}\right)^{2}$. Therefore the zero solution is not in the global attractor. We have also neglected the negative definite term $-\frac{J_{0}^{p+1}}{L^{p d}}$. By neglecting this term we lose some accuracy. However, in order to take it into account, one needs to find an accurate lower bound on $J_{0}$, namely one needs a reasonable estimate of the form $J_{0} \geq \alpha>0$. This is (notoriously) a very difficult problem! An interesting result about a lower bound on the energy for the Navier-Stokes equations on the torus is that contained in the paper [11], but unfortunately it is very large and therefore not very useful. Nevertheless it represents a very good start as a strategy for obtaining lower bounds on norms of solutions of nonlinear PDEs.

So going back to our analysis we note that $c(1)=1 \quad[9]$ and $d=1$ and by inserting the above estimate into (6) one has

$$
\bar{C}_{f} \leq L^{\frac{1}{2}}\left\langle\left(\frac{J_{2}}{J_{0}}\right)^{\frac{1}{8}}\right\rangle \leq L^{\frac{1}{2}}\left\langle\frac{J_{2}}{J_{0}}\right\rangle^{\frac{1}{8}} \leq L^{\frac{1}{2}}(2 \lambda+1)^{\frac{1}{8}} .
$$

\section{Analysis in space dimensions two}

The analysis in the two-dimensional case is similar to the one-dimensional case; one has to take into account that we are now in the two-dimensional torus $\Omega=[0, L]^{2}$. We take then

$$
\frac{1}{2} \dot{J}_{0} \leq-\frac{J_{2}}{2}+\frac{2 \lambda+1}{2} J_{0}-\left(\frac{J_{0}^{p+1}}{L^{2 p}}\right) .
$$

As in the $d=1$ case, we neglect the last term and then we divide by $J_{0}$ and then take the time average:

$$
\left\langle\frac{J_{2}}{J_{0}}\right\rangle \leq 2 \lambda+1
$$

Noting that $d=2$ and $c(2)=\sqrt{\frac{1}{\pi}}[10]$ we finally have

$$
\bar{C}_{f} \leq \sqrt{\frac{1}{\pi}} L\left\langle\left(\frac{J_{2}}{J_{0}}\right)^{\frac{1}{4}}\right\rangle \leq \sqrt{\frac{1}{\pi}} L\left\langle\frac{J_{2}}{J_{0}}\right\rangle^{\frac{1}{4}} \leq \sqrt{\frac{1}{\pi}} L(2 \lambda+1)^{\frac{1}{4}} .
$$

\section{Analysis in space dimensions three}

The analysis of the crest factor in three spatial dimensions is as follows. The spatial domain is now $\Omega=[0, L]^{3}$. We take then

$$
\frac{1}{2} \dot{J}_{0} \leq-\frac{J_{2}}{2}+\frac{2 \lambda+1}{2} J_{0}-\left(\frac{J_{0}^{p+1}}{L^{3 p}}\right) .
$$

Time averaging and applying the same strategy as in $d=1,2$ one has

$$
\left\langle\frac{J_{2}}{J_{0}}\right\rangle \leq 2 \lambda+1
$$

For the sup-norm, in three spatial dimensions we use the sharp estimate found in $[6,4]$, namely

$$
\|u\|_{\infty} \leq \sqrt{a(2 s)} \frac{\sqrt{L}}{(2 \pi)^{2}} J_{2}^{\frac{1}{2}}, \quad s=2
$$

where

$$
a(2 s)=\frac{3 b(2 s)+3 c(2 s)+d(2 s)}{2^{3-2 s}-1}
$$


with

$$
\begin{gathered}
b(2 s)=\sum^{\prime}(-1)^{k_{1}}\left(k_{1}^{2}+k_{2}^{2}+k_{3}^{2}\right)^{-s}, \\
c(2 s)=\sum^{\prime}(-1)^{k_{1}+k_{2}}\left(k_{1}^{2}+k_{2}^{2}+k_{3}^{2}\right)^{-s}, \\
d(2 s)=\sum^{\prime}(-1)^{k_{1}+k_{2}+k_{3}}\left(k_{1}^{2}+k_{2}^{2}+k_{3}^{2}\right)^{-s} .
\end{gathered}
$$

Here $k_{1}, k_{2}, k_{3}$ run all over the non zero integers.

Remark: Note that for $s=2$

$$
3 b(2 s)+3 c(2 s)+d(2 s)<0
$$

and so

$$
a(2 s)=\frac{3 b(2 s)+3 c(2 s)+d(2 s)}{2^{3-2 s}-1}>0
$$

as it should. Thus by inserting the above estimate into (6) for $d=3$ we finally obtain

$$
\bar{C}_{f} \leq\left\langle\frac{L^{\frac{3}{2}}\|u\|_{\infty}}{J_{0}^{\frac{1}{2}}}\right\rangle \leq \sqrt{a(2 s)}\left(\frac{L}{2 \pi}\right)^{2}\left\langle\left(\frac{J_{2}}{J_{0}}\right)^{\frac{1}{2}}\right\rangle \leq \sqrt{a(2 s)}\left(\frac{L}{2 \pi}\right)^{2}(2 \lambda+1)^{\frac{1}{2}} .
$$

\section{Conclusions and Open Problems}

In the previous sections we have obtained some estimates of various norms of the solutions of (a version of) the SHE. We then used these estimates to calculate their time averaged crest factor. This is defined as the ratio between the $L^{\infty}$ norm of the solution and the $L^{2}$ norm of the solution:

$$
C_{f}:=L^{\frac{d}{2}} \frac{\|u\|_{\infty}}{J_{0}^{\frac{1}{2}}}
$$

where $d$ is the spatial dimension. As we stated previously (and it is good to repeat the concept) is a dimensionless pure number and it contains important information on the "distorsion" between the "amplitude" and the $L^{2}$ norm of the solution.

Let us now discuss the implications of the estimates we have found in one, two and three space dimensions. We take into account the "pure" distortion between the sup-norm and the $L^{2}$ norm of solutions, namely we use the estimate (6). In space dimension one we found that

$$
\overline{C_{f}} \leq L^{\frac{1}{2}}(2 \lambda+1)^{\frac{1}{8}}
$$

in space dimension two we found

$$
\overline{C_{f}} \leq \frac{L}{\sqrt{\pi}}(2 \lambda+1)^{\frac{1}{4}}
$$

In space dimensions three we obtained

$$
\overline{C_{f}} \leq \sqrt{a(2 s)}\left(\frac{L}{2 \pi}\right)^{2}(2 \lambda+1)^{\frac{1}{2}} .
$$

The estimates above provide some illumination on the nature of the potential space fluctuations of the solutions of our model equation of dissipative PDE. One has just to properly "decipher" the estimates found in the various spatial dimensions.

1. In $d=1$ we found the estimate (16); in this case the crest factor scales like $\lambda^{\frac{1}{8}}$ for large $\lambda$ and scales like $L^{\frac{1}{2}}$ as a function of the length of the torus. This suggests that in one spatial dimension the dynamics of the potential fluctuations is quite "constrained" and so one can certainly say that the amount of possible turbulent behaviour is somewhat "mild".

2. In $d=2$ we found the estimate (17) and so here one can see that the crest factor scales like $\lambda^{\frac{1}{4}}$ for large $\lambda$ and scales like $L$ as a function of the length of the torus. Thus in two space dimensions we can infer that the degree of potential turbulent behaviour in space for the solutions is generally larger than in the one dimensional case both as a function of $\lambda$ and as a function of $L$. 
3. In $d=3$ is even more apparent that the space fluctuations have an even larger degree of "manoeuvre" for potential large excursions in space. In fact it $d=3$ the crest factor scales like (18), namely it scales like $\lambda^{\frac{1}{2}}$ for large $\lambda$ and it scales like $L^{2}$ as a function of the length of the torus.

By reading the above estimate it is evident that the crest factor is a proper "measure" of potential space excursions for the solutions of any dissipative PDEs. By going to higher spatial dimension the crest factor shows in a crucial way that the higher the spatial dimension, the more the space turbulent behaviour expected in solutions of PDEs. Furthermore it has to be stressed that the crest factor gives a quantitative estimate of these fluctuations, which we believe makes it even more important as an indicator for potential turbulent behaviour in solutions of dissipative PDEs. In the light of the above it would be important to compute the crest factor for other classical dissipative PDEs such as the Ginzburg-Landau equation, the Cahn-Hilliard equation and of course also for the "most" important dissipative PDEs, namely the Navier-Stokes equations. As a matter of fact we are working on the Navier-Stokes equations, and we hope to be able to provide some estimates of the crest factor for their solutions in the near future [8].

\section{References}

[1] A Frisch, Turbulence: The legacy of A. N. Kolmogorov, Cambridge University Press, Cambridge, 1996.

[2] L Berselli, T Iliescu, W Layton, Mathematics of Large Eddy Simulation of Turbulent Flows, Springer, 2006.

[3] M V Bartuccelli, J H B Deane, G Gentile, Explicit estimates on the torus for the sup-norm and the Crest Factor of solutions of the modified Kuramoto-Sivashinky Equation in one and two space dimensions. Preprint.

[4] M V Bartuccelli, Sharp constants for the $L^{\infty}{ }_{-}$norm on the torus and applications to dissipative partial differential equations. Differential Integral Equations 27 (2014), no. 1-2, 59-80.

[5] M V Bartuccelli, Explicit estimates on the torus for the sup-norm and the dissipative length scale of solutions of the Swift-Hohenberg equation in one and two space dimensions. J. Math. Anal. Appl. 411 (2014), no. 1, 166-176.

[6] M V Bartuccelli, J D Gibbon, Sharp constants in the Sobolev embedding theorem and a derivation of the Brezis-Gallouet interpolation inequality, J. Math. Phys. 52 (2011), no. 9, 093706, 9 pages.

[7] M V Bartuccelli, S A Gourley, A A Ilyin, Positivity and the attractor dimension in a fourth-order reaction diffusion equation, R. Soc. Lond. Proc. Ser. A Math. Phys. Eng. Sci. 458 (2002), no. $2022,1431-1446$.

[8] M V Bartuccelli, D Catania, On the Crest Factor for the Incompressible Two-Dimensional NavierStoke Equations on the Torus. In Preparation.

[9] A A Ilyin, Best Constants in multiplicative inequalities for sup-norms, J. London Math. Soc. $\mathbf{5 8}$ (1998), no. 1, 84-96.

[10] A A Ilyin, E S Titi, Sharp estimates for the number of degrees of freedom for the damped-driven 2D Navier-Stokes equations, J. Nonlinear Sci. 16 (2006), no. 3, 233-253.

[11] R Dascaliuc, C Foias, M S Jolly, Relations between the Energy and enstrophy on the global attractor of the 2-D Navier-Stokes equations. J. Dynam. Differential Equations 17 (2005), no. 4, 643-736.

[12] J C Robinson, Infinite-dimensional dynamical systems, Cambridge Texts in Applied Mathematics, Cambridge University Press, Cambridge, 2001.

[13] J Swift, P C Hohenberg, Hydrodynamic fluctuations at the convective instability, Phys. Rev. A 15 (1977), 319-328. 